\title{
Distance Geographic Learning Based on Collaborative Virtual Environment
}

\author{
Wenhang Li, Jianhua Gong, Daojun Wang, Mingxiang Huang, and Jieping Zhou \\ State Key Laboratory of Remote Sensing Science, \\ Institute of Remote Sensing Applications, Chinese Academy Sciences, Beijing 100101, China \\ mylihang@163.com, jhgong@sina.com.cn, \\ \{wangdaojun1980, hmx780202, janepencil\}@163.com
}

\begin{abstract}
Although collaborative e-learning has been applied in many fields, it is rarely reported in geographic education. This paper describes one prototype of collaborative virtual geographic education environment (CVGEE). The prototype, developed using Java and Java 3D, provides one immersive 3-dimensional environment and many virtual teaching methods including virtual geographic environment, virtual geographic process. Teachers and learners can discuss with each other in a social way by combination virtualization and reality. Group learning and normal teacher-learner relationship can then be realized in CVGEE. An experiment was conducted to test the functions of the CVGEE prototype.
\end{abstract}

Keywords: collaborative virtual geographic education environment (CVGEE), geography, e-learning, group learning, collaborative virtual environment (CVE).

\section{Introduction}

Education theories have been evolved from traditional teacher-centered theories to learner-centered theories and group learning theories. Collaborative E-learning (CEL) has been one of the most significant education trends recent years based on such theories, as well as virtual reality and web technologies ${ }^{[1]}$. Educations based on CEL are no longer confined to fixed place and learners, which makes public education and individual education possible. CEL always provides a friendly, immersive, 3-dimensional virtual environment and several real-time intercommunication ways for knowledge sharing and question discussion. Many CEL prototype systems have been developed, for example, INVITE ${ }^{[1]}, \mathrm{VES}^{[2]}$, VRLEARNER ${ }^{[3]}$, and other systems. Some researchers have applied CEL in various fields such as bio-education ${ }^{[4]}$, medical training ${ }^{[5]}$, and geometric spatial training ${ }^{[6]}$.

Although CEL has been applied in many subjects mentioned above, it is still rare for application in geographic education. Contrast to other subjects, geographic education needs more help from CEL, because (1) Geographic education emphasizes students' experience. But in reality, it seems impossible for teacher to take students out to all geographic environments due to funding, time, and other factors like safety. (2) The content of geographic education is complicate. Geographic knowledge covers physics, 
chemistry, mathematics and other subjects. It's a good idea for students studying together to manage these complex knowledge. New tools are suggested to help geographic education solve such problems. It is noteworthy that the innovative Google Earth has been developed and evolved rapidly ${ }^{[7]}$. Google Earth can display virtual terrain covered with remote sensing images for any place of the world. Some geography teachers have applied it in teaching. But Google Earth is not designed specifically for education, it is still necessary to explore CEL for geography.

Collaborative Virtual Geographic Education Environment (CVGEE) is such a system to help explore new ideas for geographic education. This paper introduces the prototype. Section 2 focuses on its development, including the architecture, server, and client. Section 3 presents the prototype and an experiment on tornado. Features of CVGEE, such as VGE and VGP simulation and their experience, social intercommunication, virtual teacher-learner relationship, as well as virtual teaching methods are discussed based on the experiment. Section 4 gives summary of current work, as well as prospects for future work.

\section{Development of CVGEE}

\subsection{The Architecture}

One framework of CVGEE is proposed here (Fig.1). It is based on a three-layer structure, including data layer, communication layer and representation layer. The data layer contains all resources for geography teaching and collaborations. The

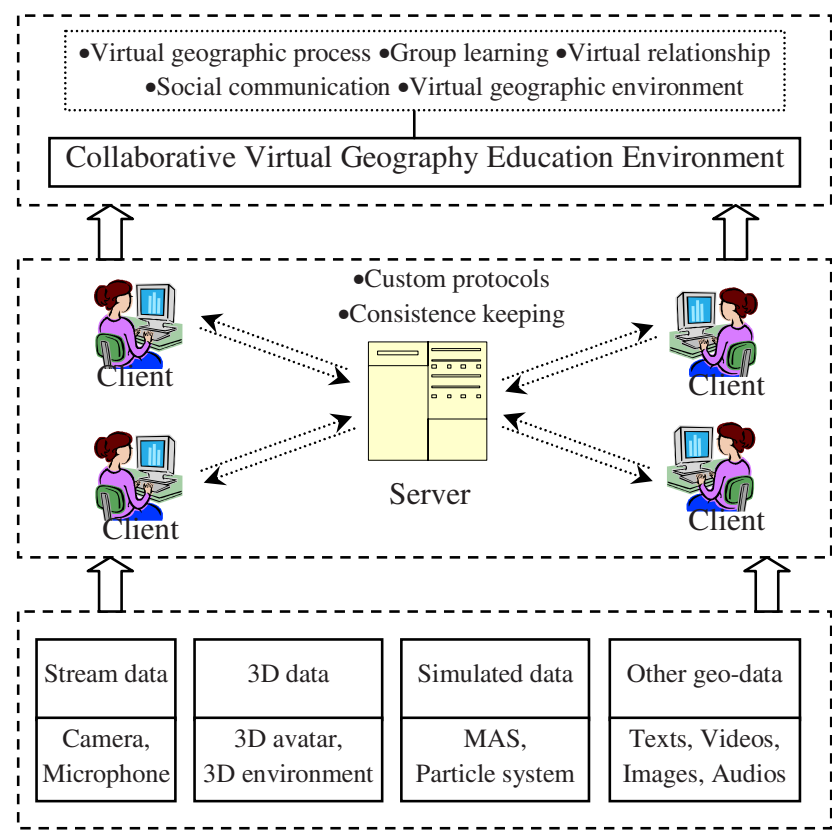

Representation layer

Communication layer

Data layer

Fig. 1. The three-layer based architecture of CVGEE 
communication layer is one $\mathrm{C} / \mathrm{S}$ mode Collaborative Virtual Environment (CVE), consisting of server and clients. Its purpose is to transfer data of the data layer, as well as collaboration messages via server to realize social communications, distance teaching, and group learning. The representations of data layer and communication layer are participants' social communication, geographic group learning, and collaborative e-learning, that is, CVGEE.

\subsection{Implementation of CVGEE}

The server is responsible for data and message transfer among clients, to keep all clients consistent. Five services, named "Message ServiceAgent", "Chat ServiceAgent", "Video ServiceAgent", "Audio ServiceAgent", and "File ServiceAgent" are deployed on the server. Each service is one multi-thread Java ServerSocket, responsible for one specific kind of data transfer. The service behavior is as follows: when new request arrives, the service will derive a new thread instance to transfer specific data. When data transfer finished, the thread will destroy itself automatically. The behavior is something like agent, so CVGEE calls these services "ServiceAgent".

Client program is the visual interface for teachers and learners to take part in the virtual education. It's a 3-dimensional virtual environment developed using Java $3 D$. The virtual scene is on a tree structure. The root node is a "SimpleUniverse" which has many child branches, termed "BranchGroup". These children branchGroups are classified according to their functions into "avatar branchGroup", "studyobject branchGroup", and "environment branchGroup". Child branchGroup may have its sub-child branchGroups. All virtual data are organized and virtual scene is then built up by this way. The virtual scene is rendered by a Canvas $3 D$ instance, who responses to the keyboard events, and menu events.

All actions of the clients are captured as commands and transferred to all others by the server. When one command arrives, the client will carry out a corresponding action to modify the virtual scene for consistency. Collaborations are then accomplished. All are done based on custom protocols (Table 1).

Table 1. Example custom collaboration protocols

\begin{tabular}{|c|c|c|c|}
\hline Action & \multicolumn{3}{|c|}{ Parameters } \\
\hline JOIN & \multicolumn{2}{|c|}{ User Name } & Avatar Location \\
\hline MOVEAVATAR & User ID & User Name & New Avatar Location \\
\hline SENDVIDEO & \multicolumn{2}{|c|}{ User ID } & Video Data \\
\hline TALK & User ID & User Name & Message Content \\
\hline
\end{tabular}

\section{Experiment of CVGEE}

\subsection{Brief Description on the Experiment}

One CVGEE prototype has been developed. As an experiment, the prototype was applied in Beijing Qianfeng middle school. The subject was on tornado, one dangerous 


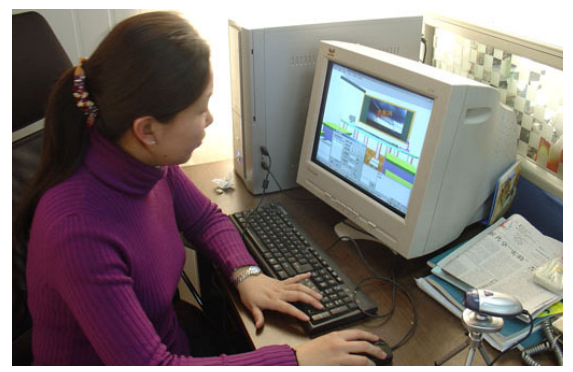

(a) teacher in experiment

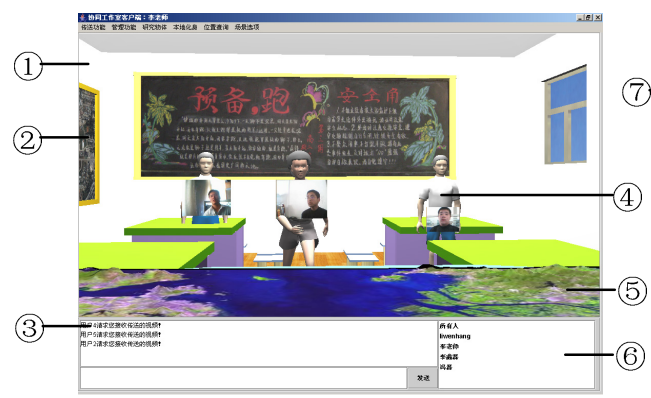

(c) snapshot of teacher's viewpoint

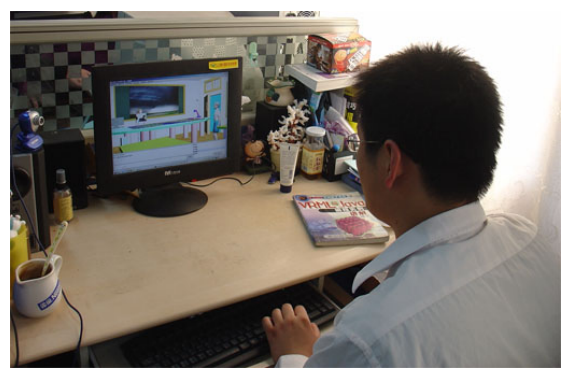

(b) student in experiment

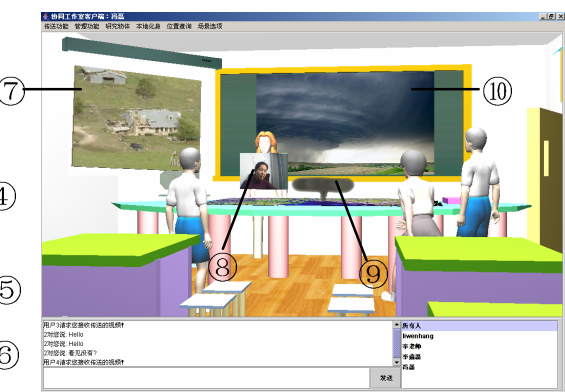

(d) snapshot of one student's viewpoint

Fig. 2. These pictures are demonstrations of the experiment. The numbers' meanings are: (1) virtual classroom, (2)remote sensing image, (3)chat text, (4)student's avatar and captured video, (5)VRML sand-table, (6)list of participants, (7)playing geo-video, (8)teacher's avatar and her captured video, (9)dynamic tornado process model (particle system), and (10)geo-slides.

geographic process. Teacher and students entered into the virtual classroom at different places via Internet. The teacher taught in voice, slides, and videos. Figure 2 gives some demonstrations of the experiment.

\subsection{VGE and VGP Simulation and Experience}

Hong Kong (Zhujiang delta) encountered with tornado was simulated during the experiment. The "terrible" event "happened" on the desk. The ground was the VRML-format Hong Kong terrain overlaid with the remote sensing image (Fig. 2 (5)). Tornado was simulated with particle system (Fig. 2 (9)). The simulation demonstrated the process that tornado came into being on the sea and moved towards the mainland.

There are two alternatives for students to experience VGE and VGP. One is to study or view as outsiders (Fig. 3 a). Another is to shrink the avatar, together with the viewpoint, into VGE or VGP to experience (Fig.3 b A). In the latter mode, participants can walk along the virtual mountains, or float across the virtual sea. It's similar to the reality. The simply changes are participants to avatars, and realistic environments to VGE and VGP. 


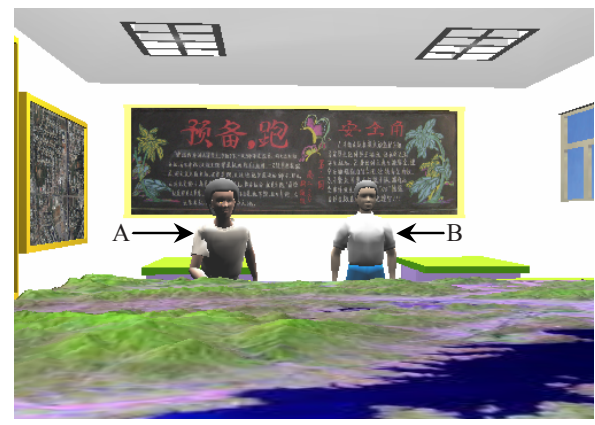

(a) outsider mode

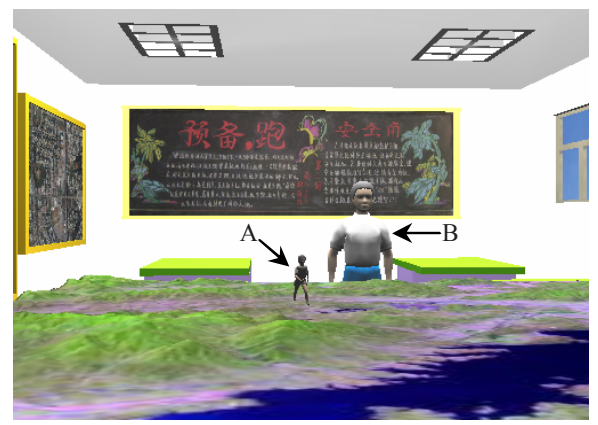

(b) experience mode

Fig. 3. VGE and VGP experience modes

\subsection{Social Intercommunication and Group Learning}

Education is one inter-communication activity between teachers and learners, which needs sight, voice, gesture, and even emotional expression to achieve mutual recognition. Referring to this mode, in the experiment, CVGEE proposed one "virtual-and-reality-mixed" method (Fig. 4 a). In virtual environment, participants have avatars. Their real figure, actions, as well as expression are captured real-time by digital cameras to "transplant" upon their virtual avatars, and their voices are broadcast through microphones. By this way, when two avatars meet in virtual classroom they (participants) can see and hear each other. Social face to face inter-communication is then simulated in CVGEE (Fig. 4 b).

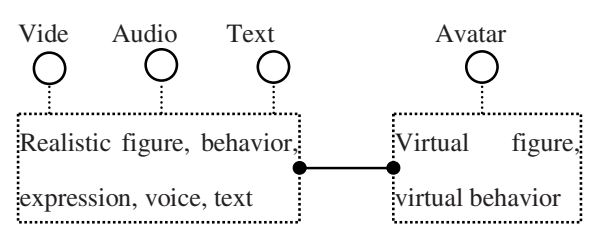

(a) communication methods

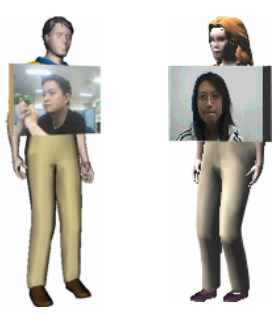

(b) face-to-face discussion

Fig. 4. Participants' realistic figures are "transplanted" on their avatars. When the avatars meet, participants can see and talk with each other. It seems nearly face-to-face communication.

Based on the above communication methods, the experiment realized group learning. They saw each other, talked with each other, or used texts to discuss on geo-materials such as slides, pictures, videos, or models. They could exchange their cognation and knowledge, or even their own geo-materials. CVGEE turned to be a platform for group discussion. 


\subsection{Virtual Teacher-Learner Relationship and Virtual Teaching Methods}

CVGEE respects learner-centered theories and group learning theories. But in the experiment, we found that most of the students, especially younger students, still could not teach themselves. So, CVGEE regards that the virtual education should not develop all by learners themselves, but follow certain supervision. So, the function of teachers should not be ignored in virtual education, and they were still the knowledge disseminator, course designers and teaching organizers. However, one significant change is teachers' status: they are not central any more but learners' helpers. Education in CVGEE is students' actively learning under guidance of teachers.

CVGEE also reserved successful traditional teaching methods in reality such as slides, geo-videos and geo-images. Students could gain geographic knowledge from various ways. Teaching methods in the experiment are list as table 2 .

Table 2. Teaching methods in CVGEE

\begin{tabular}{|c|c|c|}
\hline Classification & Features & Purpose \\
\hline Environment & 3D environment, 3D avatar & Providing one real-like education environment \\
\hline Virtual simulations & VGE,VGP & $\begin{array}{l}\text { Achieving virtual experience of geographic } \\
\text { environment and geographic process }\end{array}$ \\
\hline Traditional methods & Slides, video, audio, images & $\begin{array}{l}\text { Inheriting the successful realistic education } \\
\text { methods }\end{array}$ \\
\hline $\begin{array}{l}\text { Group learning } \\
\text { methods }\end{array}$ & $\begin{array}{l}\text { Captured video, captured } \\
\text { audio, text chat }\end{array}$ & $\begin{array}{l}\text { Simulating virtual face-to-face } \\
\text { inter-communication }\end{array}$ \\
\hline
\end{tabular}

\section{Conclusion and Future Work}

In this paper, we discussed the collaborative virtual geographic education environment and its prototype. Contributions of this paper to geographic education can be summarized as following: (1) CVGEE turns geographic education into the virtual one based on a distributed collaborative virtual environment. Distance teaching, distance learning and group learning can be carried out in CVGEE; (2) CVGEE helps solve the puzzle of experience by introducing virtual geographic environments and virtual geographic processes; (3) CVGEE appreciates socially face-to-face participants' communication in virtual education activities; and (4) CVGEE indicates a normal virtual teacher-learner relationship for virtual geographic education.

But further contributions can be achieved in future work, not only for geographic education but also for education itself: (1) Educations have been carried out in virtual space. Since present education theories are based on realistic education behaviors, then one question should be asked: are they fit for the virtual ones? How about the relationship between teachers and learners in the virtual world? They're rarely seen in literature. Maybe new theories should be proposed. (2) One more thing needs to be pointed out that virtual educations including CVGEE are not substitute for the realistic ones. They are supplements for each other. So it will be interestingly innovative to integrate the virtual education with the realistic one. Nevertheless, it is a worthwhile field of study CVGEE for geographic education. 


\section{Acknowledgements}

This research is partially supported by the National High-tech R\&D Program (863 Program) No. 2006AA12Z204 and the Knowledge Innovation Program of the Chinese Academy of Sciences, Grant No. Kzcx2-yw-126-01.

\section{References}

1. Kuljis J.: A Distributed Virtual Collaborative Learning Environment. In Proceedings of the IASTED International Conference, Applied Informatics, Innsbruck, Austria (2002) 464-469

2. Bouras C., Fotakis D., Kapoulas V.,Koubek A., Mayer H., Rehatscheck H.: Virtual European School-VES. In Proceedings of the IEEE Multimedia Systems'99, Special Session on European Projects, Florence, Italy (1999) 1055-1057

3. Kladias N., Pantazidis T., Avagianos M., Kalamas I., Kopidakis Y., Labbri M.: The VRLEARNERS project: development of a Virtual Reality learning environment providing access to digital museums. http://www.vrlearners.iis.gr/vrlearners/vrlearn.RTF

4. Okada M., Tarumi H., Yoshimura T.: Distributed virtual environment realizing collaborative environment education. In Proceedings of the 2001 ACM symposium on Applied computing, Las Vegas, USA (2001) 83-88

5. Bernhard J., Abdul A., Matthias W.: The Affective Virtual Patient: An E-Learning Tool for Social Interaction Training within the Medical Field. http://isnm.de/ aahad/Downloads/ AVP_TESI.pdf

6. Liu D., Wang T. M.: A virtual reality training system fro robot assisted neurosurgery. In Advances in Artificial Reality and Tele-Existence, ICAT 2006, Hangzhou, China (2006) 457-466

7. Declan B.: Virtual globes: The web-wide world. Nature, 439(16) (2006) 776-778 